\title{
Advanced sensor fault detection and isolation for electro-mechanical flight actuators
}

\author{
Daniel Ossmann and Franciscus L. J. van der Linden \\ Institute of System Dynamics and Control \\ German Aerospace Center (DLR) \\ Oberpfaffenhofen, Germany \\ Emails: \{daniel.ossmann, franciscus.vanderlinden\}@dlr.de
}

\begin{abstract}
Moving towards the more electric aircraft to be able to replace mechanic, hydraulic and pneumatic components of an aircraft, the aircraft industry calls for new technologies able to support this trend. One of these technologies is the development of advanced electro-mechanical actuators for aircraft control surfaces. Step by step hydraulic actuators are replaced by their electro-mechanical alternatives featuring weight and cost savings. As hydraulic actuators are used for decades by the aircraft industry, they are augmented with advanced signal and model based fault detection and diagnosis systems able to monitor the actuator and initiate adaptations in case of failures. For electromechanical actuators such advanced monitoring systems are still in the initial stages. In this paper, fault detection and isolation filters are designed by applying advanced residual filter synthesis algorithms to be able to monitor the sensor of electro-mechanical actuators. This paves the way for possible adaptations in electromechanical actuator systems in case of failures.
\end{abstract}

Keywords-Fault detection and isolation, fault tolerance, flight actuators.

\section{INTRODUCTION}

The aircraft industry relies on hydraulic actuation systems to provide adequate system performance and stability and traditionally uses physical redundancy of these systems to increase system autonomy. Such hydraulic systems lead to an increased aircraft weight, which becomes more and more problematic when trying to satisfy the greener imperatives demanded by the society. Hence, hydraulic components are replaced step-by-step by their lighter electrical alternatives, moving the aircraft industry towards the more electric aircraft.

Today's hydraulic flight actuators feature advanced signal and model based fault detection and diagnosis (FDD) systems. If any fault is detected on a hydraulic actuator by these FDD systems, the actuator is set to passive mode and a redundant actuator is activated and takes over. The use of advanced FDD systems specially supports the detection of low amplitude faults, leading to two main positive effects lowering the aircraft operational costs: (i) suboptimal operation of the aircraft with an increased drag due to unwanted deflections of the control surface caused by faults and leading to an increased fuel burn can be avoided; (ii) the aircrafts' structural design margins can be lowered automatically decreasing aircraft weight and operational costs [1]. The reason for this is that these margins are defined by the largest fault amplitudes, which cannot be detected by the FDD system of the aircraft but potentially can excite the aircraft structural modes if they occur. Thus, lower

978-1-4673-7501-6/15/\$31.00 (C)2015 IEEE detectable fault amplitudes lead to lower structural design margins. Taking the possibilities of cost savings using electromechanical actuators (EMAs) together with advanced FDD algorithms into account, indicates the future trend of using electro-mechanical actuators together with reliable FDD systems to monitor the EMAs. However, while literature regarding FDD approaches for hydraulic flight actuators is rich, fewer contributions can be found for EMAs.

The detection of faults in classical hydraulic flight actuators is an intensively studied topic in literature. Advanced methods as LPV techniques in [2] and [3], sliding mode observers in [4], nonlinear observer based approaches in [5] and $H_{\infty}$ techniques in [6] have been applied to monitor hydraulic actuator systems of modern aircraft locally, i.e., by looking at the input-output dynamics of the actuator. System wide monitoring approaches using the input-output behavior of the overall aircraft dynamics are provided by [7] and [8]. [9] proposes a hybrid system combining local and system wide fault detection approaches. While various approaches are able to detect faults on actuators quite well, none of them tries to isolate the different faults, which can occur for example at the mechanical parts of the actuator itself as well as on the actuator's sensors, required to close the actuator's control loop(s). In [10] an enhanced FDD system including fault isolation is proposed for hydraulically flight actuators to close this gap

For the fault monitoring of EMAs in [11]-[13] signal processing based approaches are proposed, however, in none of them fault isolation issues are discussed. In [14] an approach based on online parameter identification is discussed, also taking fault isolation aspects based on statistical characteristics of the identified parameters into account. The method includes the solving of a non-convex optimization problem for parameter identification as well as a statistical analysis to monitor an actuator. The monitoring of all actuators of a modern civil aircraft with such an method seems to be rather ambiguous when taking into account the limited computational power on aircraft systems in combination with the number of actuators, which can exceed 20 easily for modern augmented aircraft configurations. Additionally, it has to be noted, that all the electrical (e.g. motor) and mechanical components (e.g. gear) of the EMA need to be monitored by dedicated health monitoring algorithms able to detect very small performance degradations, enabling a replacement of the actuators before abrupt faults can occur. Alternatively, fault tolerant subcomponents in an EMA as proposed in [15] can be used. The application of one of these options is necessary as in contrast to hydraulic actuators 
state-of-the-art EMAs cannot be set to passive mode to avoid any unwanted deflections of the control surface.

Thus, fault detection for the mechanic and electronic components of the EMA is not of primary interest, as there are no adaptation options available and faults on these components need to be predicted before their occurrence. However, the usage of model based FDD systems for EMAs can support the detection of possible sensor malfunctions to decrease necessary sensor redundancy [15] on the actuator as well as detect low sensor fault amplitudes which could excite the aircraft structural modes or lead to a suboptimal operation of the aircraft. EMAs commonly use cascade control algorithms requiring a set of dedicated sensors. Abrupt faults on these sensors may lead to the described effects. In this paper a closer look on the ability to monitor the EMA's sensor system and the possibility to detect and isolate different sensor faults is given. The isolation information can be used to adapt the actuator in case of sensor faults, for example by switching to a redundant (physical or virtual) sensor or even accommodate the fault.

The design of the fault detection and isolation (FDI) filters in this paper relies on recently developed linear tools to solve the fault detection and isolation problem. The tools make extensive use of numerically stable algorithms to determine the left nullspace basis of a rational transfer matrix, thereby determining detectors of minimal order. Section II describes the general FDD problem formulation for the EMA and discusses the proposed steps for the design and validation of the EMA FDD system. In section III, a model of an electro-mechanical aircraft actuator is derived, including the modeling of relevant sensor faults. A linear model of the actuator description is presented enabling the design of linear residual filters. In section IV the basics of the nullspace-based fault detection and isolation residual filter design procedure and its application to the EMA model is presented. In section $\mathrm{V}$ the validation of the FDD system using a nonlinear high fidelity actuator benchmark model is shown.

\section{PROBLEM FORMULATION}

By applying nullspace computation based synthesis tools a linear FDI filter shall be designed, able to detect and isolate different sensor faults on the EMA. The EMA consists of an electric motor which generates via a gear with two stages a (longitudinal) displacement of a ball-screw. The sensors of the EMA measure different states of the electric motor and the ball screw, a linear model of the motor, the gear and the ball screw serves as synthesis model for the residual filter, as depicted in Fig. 1. The green lines show the available signals, either measured or computed by the actuator's controller. The EMA is connected to an aircraft control surface on which an aerodynamic load acts on. This aerodynamic load is transferred via the housing and the surface to the gear, generating the actuator's load input. Thus, the blocks in Fig. 1 are connected via feedback loops with each other.

The residual filter to be designed may use all available signals (measured or computed) to generate a residual signal vector. In the fault free case its residual signals shall be equal zero (or sufficiently small), requiring the decoupling of any inputs and disturbances from the residuals. In the different fault scenarios the residuals shall generate a unique signature

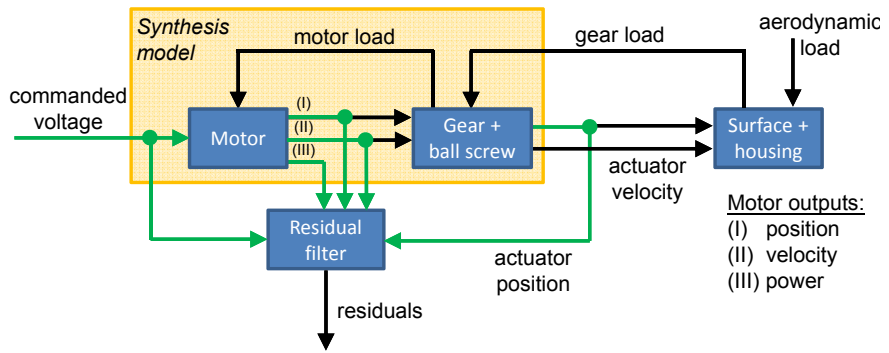

Fig. 1. Block diagram of the residual filter synthesis model of the actuator

for each fault enabling the detection and isolation of the faults. The employed model based methodology for the residual filter design has the following steps:

1) Development of suitable linear synthesis models of the underlying EMA.

2) Synthesis of a residual generator for fault detection and isolation.

3) Validation of robustness and detection performance of the generated filter using a nonlinear closed loop benchmark model of the actuator.

These steps are described and discussed in more detail in the following sections.

\section{ELECTRO-MECHANICAL ACTUATOR MODELLING}

In this section the nonlinear modelling and the derivation of the linear residual filter synthesis model of the EMA are discussed. The EMA driving the control surface consists of an electric motor, a gear with two stages and a ball screw. As all sensors are placed on these parts of the EMA, a linear open loop synthesis model will be derived describing the dynamical behavior of these elements. For realistic simulation and validation purposes the EMA is connected to a housing and linked to an aircraft control surface. The overall system is operated with a cascade controller featuring motor current, motor velocity and actuator position feedbacks as depicted in Fig. 2. Additionally, the motor position is available as measured signal. As mentioned in the last section, the final FDD algorithm uses all measurement signals as well as the controller input to generate the residual vector.

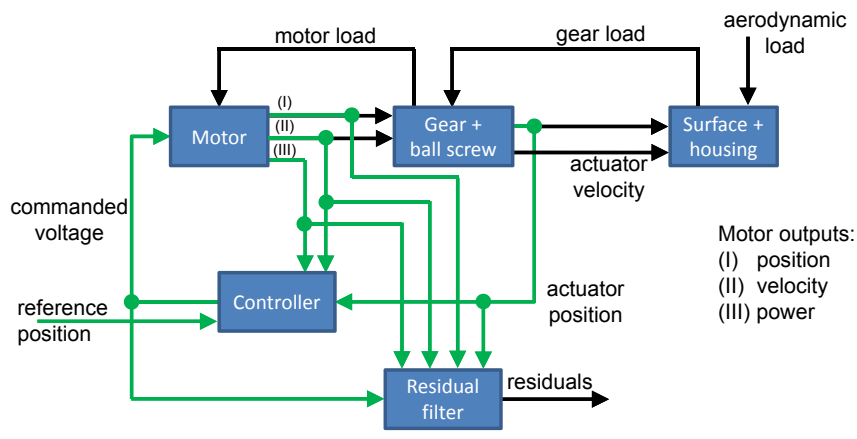

Fig. 2. Block diagram of the closed loop nonlinear simulation model augmented with controller and FDD system 


\section{A. Electric motor}

The nonlinear model of the motor is described by the differential equation of the motor current

$$
\dot{I}_{M}=k_{1}^{-1}\left(-\dot{\phi} k_{u}\left(I_{M}\right)-k_{2} I_{M}+U\right),
$$

where $k_{u}\left(I_{M}\right)$ is the current dependent motor parameter, $U$ the input voltage, $k_{1}$ the inductance, $k_{2}$ the motor resistance constant and $\dot{\phi}$ the angular velocity. The differential equation for the angular acceleration is given by

$$
\ddot{\phi}=J_{M}^{-1}\left(-k_{l m} \dot{\phi}+k_{v} k_{u}\left(I_{M}\right) I_{M}+k_{c 1} \sin \left(k_{c 2} \phi\right)-M_{e}\right),
$$

where $J_{M}$ is the motor's moment of inertia, $k_{l m}$ the gain to describe viscous losses, $k_{v}$ the transfer gain from current to generated moment, $k_{c 1}$ and $k_{c 2}$ the amplitude and phase of the periodic mechanical losses due to the cogging in the motor and finally, $M_{e}$ the acting torque load.

Linear Model: For the linearization of the electric motor two simplifications are introduced: the gain $k_{u}\left(I_{M}\right)$ is set to an approximated constant value; in (2) the influence of the mechanical losses due to the motor cogging are neglected. Taking these two simplifications into account the equations can be written as linear third order state space system

$$
\begin{aligned}
\dot{x}_{M} & =A_{M} x_{M}+B_{M} u_{M} \\
y_{M} & =x_{M},
\end{aligned}
$$

with the state vector $x_{M}^{T}=\left[\begin{array}{lll}\phi_{M} & \dot{\phi}_{M} & I_{M}\end{array}\right]$, the input vector $u_{M}^{T}=\left[\begin{array}{ll}U & M_{e}\end{array}\right]$ and the output vector $y_{M}$. The state space matrices $A_{M}$ and $B_{M}$ are given by

$$
\begin{aligned}
A_{M} & =\left[\begin{array}{ccc}
0 & 1 & 0 \\
0 & 0 & k_{v} k_{u} / J_{M} \\
0 & -k_{u} / k_{1} & -k_{2} / k_{1}
\end{array}\right], \\
B_{M} & =\left[\begin{array}{cc}
0 & 0 \\
0 & -1 / J_{M} \\
1 / k_{1} & 0
\end{array}\right] .
\end{aligned}
$$

\section{B. Gear and ball screw modelling}

The main mechanical part of the actuator consists of two gear stages and a ball screw. The two gear stages as well as the ball screw are modelled as linear second order mass-springdamper elements which are augmented with nonlinear effects as breakaway forces, backlash, dependencies of the transfer effectiveness on the load direction as well as drag induced by the ball screw. For the derivation of the linear approximation these effects are neglected and thus, are not described in more detail. However, they are included in the nonlinear simulation model used for validation purposes of the designed residual generator. The linear second order mass-spring-damper model for the $i^{t h}$ model element, where $i=1$ and $i=2$ correspond to the two gear stages and $i=3$ to the ball screw, is given by

$$
\begin{aligned}
\ddot{\phi}_{i}= & 1 / J_{i}\left(d_{i}\left(\dot{\bar{\phi}}_{i-1}-\dot{\phi}_{i}\right)-d_{f, i} \dot{\phi}_{i}\right. \\
& \left.+c_{i}\left(\bar{\phi}_{i-1}-\phi_{i}\right)-n_{i}^{-1} M_{L, i+1}\right) .
\end{aligned}
$$

where $\phi_{i}$ and $\dot{\phi}_{i}$ are the rotation angle and angular velocity of the $i^{t h}$ model element defining the state vector and $\bar{\phi}_{i-1}$ and $\dot{\bar{\phi}}_{i-1}$ are the rotation angle and angular velocity of the $(i-1)^{t h}$ model element defining the input vector. $J_{i}$ is the corresponding moment of inertia, $d_{i}$ the damping coefficient, $n_{i}$ the transfer ratio, $c_{i}$ the stiffness and $M_{L, i+1}$ the load input. $d_{f, i}$ is used to describe the linear approximation of friction effects. The output equation of each stage is defined by

$$
\left[\begin{array}{c}
\bar{\phi}_{i} \\
\dot{\bar{\phi}}_{i} \\
M_{L, i}
\end{array}\right]=C_{g, i}\left[\begin{array}{c}
\phi_{i} \\
\dot{\phi}_{i}
\end{array}\right]+D_{g, i}\left[\begin{array}{c}
\phi_{i-1} \\
\dot{\phi}_{i-1} \\
M_{L, i+1}
\end{array}\right],
$$

where

$$
C_{g, i}=\left[\begin{array}{cc}
1 / n_{i} & 0 \\
0 & 1 / n_{i} \\
-c_{i} & -d_{i}
\end{array}\right]
$$

and

$$
D_{g, i}=\left[\begin{array}{ccc}
0 & 0 & 0 \\
0 & 0 & 0 \\
c_{i} & d_{i} & 0
\end{array}\right] .
$$

to allow an adequate connection of the three stages: the outputs $\bar{\phi}_{i}$ and $\dot{\bar{\phi}}_{i}$ of the $i^{t h}$ model element are the inputs of the $(i+$ $1)^{t h}$ stage, while the third output of each stage, namely the load $M_{L, i}$, is fed back to the $(i-1)^{t h}$ model element; the inputs of the first gear stage is defined by the angular position and velocity of the electric motor and the load of the ball screw is calculated by $M_{L, 3}=-F_{a c t} n_{3}^{-1}$, where $F_{a c t}$ is the actuator load. The exact definition of the actuator load is described in more detail later, but it is important to note that beside the input voltage, this load defines the second input of the overall linear motor-gear model. However, this input cannot be measured and has to be treated as disturbance during the design process. Further, it is important to note that the transfer ratio $n_{3}$ includes the transition from angular postilion and velocity of the screw into its longitudinal displacement. This displacement is defined as the displacement of the actuator in meters: $x_{A}=\bar{\phi}_{3}=$ $n_{3}^{-1} \phi_{3}$ and $\dot{x}_{A}=\dot{\bar{\phi}}_{3}=n_{3}^{-1} \bar{\phi}_{3}$ respectively.

\section{Residual filter synthesis model}

The overall linear model of the motor, the gear and the ball screw are connected with each other leading to a linear $9^{\text {th }}$ order model of the form

$$
\begin{aligned}
\dot{x} & =A x+b_{u} U+b_{d} M_{L, 3} \\
y & =C x,
\end{aligned}
$$

where $b_{u}$ is the input vector and $b_{d}$ the input disturbance vector which are multiplied with the commanded voltage $U$ and actuator load $M_{L, 3}$, acting as disturbance on the system. The elements of the measured output vector $y=\left[\begin{array}{ll}\phi_{M} & \dot{\phi}_{M} I_{M} x_{A}\end{array}\right]^{T}$ are the motor position, its velocity and current as well as the actuator position (screw displacement). The state space matrices $A$ and $C$ are not described in more detail due to space constraints of the paper. Fig. 3 shows a simulation based comparison of the nonlinear model and its linear approximation using two impulse-like excitations applied on the motor voltage demand and the aerodynamic hinge moment. Note that the first and third diagram of Fig. 3 are normalized. The three depicted outputs of the linear model match quite well to the outputs of the nonlinear model, indicating a satisfactory approximation of the nonlinear actuator dynamics.

For the residual filer synthesis the linear model (9) needs to be enhanced by sensor fault inputs. Faults on the sensors 

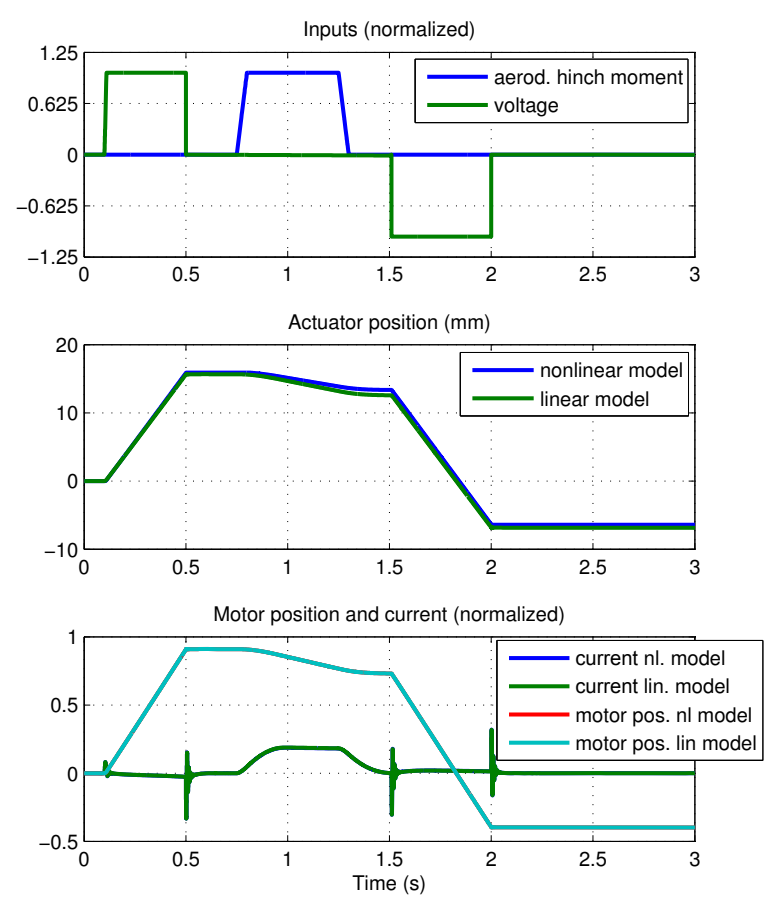

Fig. 3. Comparison of the nonlinear model and its linear approximation by open loop simulations

of motor velocity, motor current and actuator position are considered and modelled as additive faults in the output equation of (9), leading to the redefined output equation

$$
y=C x+f
$$

of the synthesis model, where the elements of the fault vector $f=\left[\begin{array}{llll}0 & f_{1} & f_{2} & f_{3}\end{array}\right]^{T}$ are the three fault signals acting on motor velocity, motor current and actuator position. Due to the definition of the faults as generic input signals, the synthesis approach does not limited the designed filter to the detection and isolation of a specific type of fault (loss of efficiency, bias, sensor drift, etc.). For validation purposes a small sensor bias will be considered which is usually harder to detect than any fault which changes its amplitude over time (e.g., sensor drift).

Order reduction techniques have been applied to further reduce the order of the linear motor-gear model, ending up with a $3^{\text {rd }}$ order model. This model serves as synthesis model for the residual filter design. Such a low order approximation supports the design of low order residual filters minimizing implementation costs on the final hardware.

\section{Elastic modelling of control surface and housing}

The model of the housing and the control surface is part of the nonlinear benchmark model used to validate the designed residual generator. The housing is modelled as second order differential equation of the form

$$
\ddot{x}_{H}=-m_{H}^{-1}\left(d_{H} \dot{x}_{H}+c_{H} x_{H}+F_{a c t}\right),
$$

where $x_{H}$ is the housing position, $m_{H}$ its mass, $d_{H}$ the housing damping coefficient, $c_{H}$ its stiffness and $F_{a c t}$ the actuator load acting on the housing. The dynamics of the control surface are more complex and modelled by

$$
\ddot{\phi}_{S}=J_{S}^{-1}\left(M_{\text {aero }}+M_{d}+F_{a c t} l\left(x_{A}\right)\right),
$$

where $\ddot{\phi}_{S}$ is the angular acceleration of the surface, $M_{a e r o}$ the aerodynamic load moment, $M_{d}$ the damping moment and $l$ the effective lever arm, depending nonlinearly on the actuator displacement $x_{A}$. The load $F_{a c t}$ is given by

$$
F_{a c t}=c_{S}\left(x_{A}-l\left(x_{A}\right) \phi_{S}+x_{H}\right),
$$

where $c_{S}$ describes the stiffness of the surface. The damping moment $M_{d}$ is a highly nonlinear differential equation which is not stated at this point due to confidential reasons. As for the residual filter synthesis these parts of the system are not relevant, no linear models need to be derived. However, they are part of the nonlinear benchmark model used to validate the designed FDD system.

\section{FDI FILTER SYNTHESIS FOR THE EMA}

In this section the linear synthesis techniques which can be used to solve the EMA sensor fault detection and isolation problem are described. The three EMA sensors to be monitored are the sensors used for control purposes, namely the actuator position, motor velocity and motor current. The signals to be used by the residual generator are these three sensor signals, the available measurement of the motor position as well as the commanded input voltage. The load acting on the gear is assumed to be not measurable and is therefore treated as unknown disturbance which has to be decoupled from the filter outputs. To be able to apply the nullspace based residual filter synthesis procedure some transformations of the derived equations of the EMA are necessary:

The linear description (9) and (10) of the EMA can be written in input-output form

$$
\mathbf{y}(s)=G_{u}(s) \mathbf{U}(s)+G_{d}(s) \mathbf{M}_{L, 3}(s)+G_{f}(s) \mathbf{f}(s),
$$

where $\mathbf{y}(s), \mathbf{U}(s), \mathbf{M}_{L, 3}(s)$ and $\mathbf{f}(s)$ are the Laplacetransformed quantities of the systems measurement signals $y(t)$, the control input voltage $U(t)$, the actuator load $M_{i, 3}(t)$ and the fault vector $f(t)$, respectively. $G_{u}(s)$ and $G_{d}(s)$ are the corresponding $4 \times 1$ transfer matrices and $G_{f}$ is a constant $4 \times 3$ matrix, as the failures directly influence the outputs $y(s)$. To solve the fault detection and isolation problem for the system described by (14) the residual generator

$$
\mathbf{r}(s)=Q(s)\left[\begin{array}{c}
\mathbf{y}(s) \\
\mathbf{U}(s)
\end{array}\right]
$$

shall be generated, which uses the available input $\mathbf{U}(s)$ and outputs $\mathbf{y}(s)$ of the system to generate a residual vector. In (15) $\mathbf{r}(s)$ is the Laplace-transformed quantity of the residual vector $r(t)$. The residuals shall be zero in fault free situations while generating a unique signature for each fault. For a physically realizable filter, $Q(s)$ must be proper and stable, having only poles with negative real parts. 


\section{A. Isolateablity of faults}

To gain knowledge if the different faults can be isolated from each other and the corresponding residual filter can be designed requires the understanding of the fault signature matrix (FSM). The FSM $S$ of a system augmented with a residual generator describes the effects of the faults on the residuals. As presented in [16] and applied in [10] it is possible to determine the achievable fault signature matrix of the underlying system before the actual synthesis of the residual filter, select an adequate desired fault signature matrix $\bar{S}$ and use this matrix as input for the design of the residual filter. Let $R_{f_{i}}^{j}(s)$ be the transfer function of the $i$-th fault to the $j$-th residual. Then the entries of $S$ are defined as

$$
S_{j, i}= \begin{cases}1 & \text { if } R_{f_{i}}^{j}(0) \neq 0 \\ -1 & \text { if } R_{f_{i}}^{j}(0)=0 \text { and } R_{f_{i}}^{j}(s) \neq 0 \\ 0 & \text { if } R_{f_{i}}^{j_{i}}(s)=0 .\end{cases}
$$

In words, the entry ' 1 ' indicates a stationary influence of the fault to the residual, the entry ' 0 ' indicates decoupled behavior of the residual from the fault, and the entry ' -1 ' indicates that the residual is influenced by the fault, but its steady state value is 0 . The desired FSM of a residual filter is always the identity matrix with the dimension equal to the number of faults. Such a FSM solves the so called fault detection and strong isolation problem: each residual is influenced by only one fault, thus directly indicating the occurrence or absence of a certain fault. This ensures fault isolation also in multiple fault situations, which is not possible with other structures of the FSM, just solving the fault detection and weak isolation problem.

The calculation of the FSM for the linear EMA model reveals that fault detection together with strong fault isolation is possible. The corresponding strong fault detection and isolation problem can be defined as follows: Determine a physically realizable linear residual generation filter of the form (15) having three outputs and least dynamical order such that:

(a) $\quad r(t)=0$ when $f_{i}(t)=0 ;$ for $i=1,2,3$

(b) $\quad r_{i}(t) \neq 0$ when $f_{i}(t) \neq 0$; for $i=1,2,3$

(c) all residuals are asymptotically bounded.

(a) requires all the residuals to be zero in any fault free situation, while $(b)$ requires a steady state response of the residual $i$ if the fault $i$ occurs. (c) demands steady state responses to constant faults.

\section{B. Nullspace based FDI filter synthesis}

One possible approach to solve the described problem is to apply the nullspace method. This method has the advantage that it enables the direct decoupling of both, disturbances and inputs, and provides residual filters of minimal order, which minimizes implementation costs on the final hardware. The principals of the method are described using the linear EMA model below: First, (14) is inserted into (15), leading to

$$
r(s)=Q(s)\left[\begin{array}{ccc}
G_{u}(s) & G_{d}(s) & G_{f} \\
1 & 0 & 0
\end{array}\right]\left[\begin{array}{c}
\mathbf{U}(s) \\
\mathbf{M}_{L, 3}(s) \\
\mathbf{f}(s)
\end{array}\right]
$$

For each fault $i$ to be detected the decoupling condition

$$
Q^{i}(s)\left[\begin{array}{ccc}
G_{u}(s) & G_{d}(s) & \tilde{G}_{f}^{i} \\
1 & 0 & 0
\end{array}\right]:=Q^{i}(s) G_{e}(s)=0
$$

with $i=1,2,3$, needs to be fulfilled [17]. In (19) $\tilde{G}_{f}^{i}$ is the redefined fault transfer matrix, formed from the columns of $G_{f}$ with the indices $j \neq i$. In words, (19) demands a residual filter $Q^{i}(s)$ which decouples the inputs $u$, the disturbance $d$ and the faults with the indices $j \neq i$ from the residual $i$. The problem of finding such a $Q^{i}(s)$ can be solved by determining a left nullspace basis of $G_{e}(s)$. Numerically stable methods to determine minimal rational left nullspace bases of $G_{e}(s)$ are presented in [17].

Each row of such a nullspace basis as well as each linear combination of its rows fulfills the decoupling condition (19) and is a potential candidate for $Q^{i}(s)$. However, beside solving the decoupling condition $(a)$ of the FDI problem (17), the nullspace basis has to include basis vectors directly coupling the fault $i$ to the output to be able to fulfill $(b)$. If such basis vectors are not available, no analytical solution to the FDI problem exists [17]. If such a basis vectors are available, $Q^{i}(s)$ can be determined as any linear combination of the basis vectors which fulfills $(b)$ and the minimal order constraint. $(c)$ can always be fulfilled as the dynamics of the filter can be freely chosen.

With the described approach for each fault $i$ of the EMA a residual filter $Q^{i}(s)$ is designed separately and assembled to one residual generator at the end according to

$$
Q(s)=\left[\begin{array}{lll}
Q^{1}(s) & Q^{2}(s) & Q^{3}(s)
\end{array}\right]^{T} .
$$

\section{Characteristics of the EMA residual generator}

The filter $Q^{1}(s)$ to detect faults on the actuator position sensor is a constant filter, which compares the actuator position with the motor position multiplied by a transfer gain. The other two filters $Q^{2}(s)$ and $Q^{3}(s)$ to monitor the motor velocity and the motor current sensors are both first order filters. This results in a second order residual generator when assembling the three filters together as described by (20). This minimal order filter design is one major advantage of the nullspace based synthesis method compared to other approaches. As stated in [18], minimal filters tend to be more robust against model uncertainties than non-minimal filters. Further, the implementation and computation costs of the filter on the final hardware are minimized. Another advantage of the method is, that the dynamical behavior of the residual filter, and thus, the transfer behavior of the faults to the residuals, can be freely chosen. In the example in this paper, the two poles of the filter have been set to a value of -10 , allowing the detection of the faults in reasonable time but still filtering out high frequency measurement noise in the residual.

\section{FILTER ASSESSMENT}

The designed residual generator is validated using a high fidelity nonlinear benchmark model of the EMA. The simulation model features a cascade controller including actuator position, motor speed and motor current control. For the fault free testing a realistic trajectory of the reference voltage and the aerodynamic load acting on the control surface is selected. For the simulations with faults a sensor bias type of fault is chosen. The fault amplitudes to be detected are $2 \mathrm{~mm}$ for the actuator position sensor, $25 \mathrm{rad} / \mathrm{s}$ for the motor velocity sensor and $6 \mathrm{~A}$ for the motor current sensor. These correspond to about $5 \%$ to $10 \%$ of the normal operational range of the measured 
parameters. We assume that these values have a noticeable impact on the aircraft dynamics and have to be detected while values below can be handled robustly by the controller.

\section{A. Fault free simulations}

The fault free simulation of the EMA is used to prove that the residual generator works adequately during fault free operation, i.e., the residual signals are sufficiently small. Due to model uncertainties and approximation errors during the development of the linear model the residuals will not be equal zero when the residual generator is operated on the real system. Thus, a threshold needs to be set in the dedicated decision logic to differentiate between fault free and faulty behavior. In the first diagram of Fig. 4 the demanded position trajectory is depicted, including various commanded amplitudes and driving speeds. In the second diagram the defined aerodynamic hinge moment which acts as disturbance is depicted, while in the third diagram the resulting actuator load directly acting on the motor-gear system is plotted. Finally, the second diagram shows the commanded input voltage by the controller which is propagated to the electric motor.

Fig. 5 shows the three residual signals during the simulation. As expected the residuals are not equal to zero during the simulation of the linear fault detection filter using the high fidelity nonlinear benchmark model. However, they stay below the fault amplitudes to be detected allowing the selection of adequate thresholds. The residual $r_{3}$ comes closer to the fault amplitude to be detected than the other two residuals. While the maximum is still acceptable its dynamic influence can cause problems when trying to estimate fault amplitude exactly.

\section{B. Fault simulations}

To check the ability of the residual generator to detect and isolate faults, simulations with faults are conducted using the nonlinear benchmark model. To have a comparable evaluation the faults occur during the same maneuver which was used for the fault free evaluation. Fig. 6 shows the residual signals during the fault simulations. Note, that three dedicated simulations have been conducted, each with a different fault. In Fig. 6 only the corresponding residual which needs to be excited due to the fault is depicted, as the other two residuals stay sufficiently small not causing any false alarms as it has been shown by the fault free simulations. In all three simulations the fault occurs at a simulation time of $1 \mathrm{~s}$. In each case the residuals exceed correctly their defined thresholds. These thresholds have been set taking the maximum excitation of the residuals in the fault free situations as well as the fault amplitudes to be detected into account. In case of faults on the actuator position sensor and the motor velocity sensor the faults are tracked very well, however not perfect. This is the result of the residuals being influenced by the command inputs and disturbance which was demonstrated by the fault free simulations. The same reasons lead to several threshold undershoots of the residual used to monitor the motor current. This was expected from the results of the fault free simulations. As only one exceeding of the threshold is required to isolate the fault, this is manageable for detection and isolation purposes. The dynamical behavior of the three residuals exactly reflects the designed dynamics. While the first filter is a constant filter and the fault directly
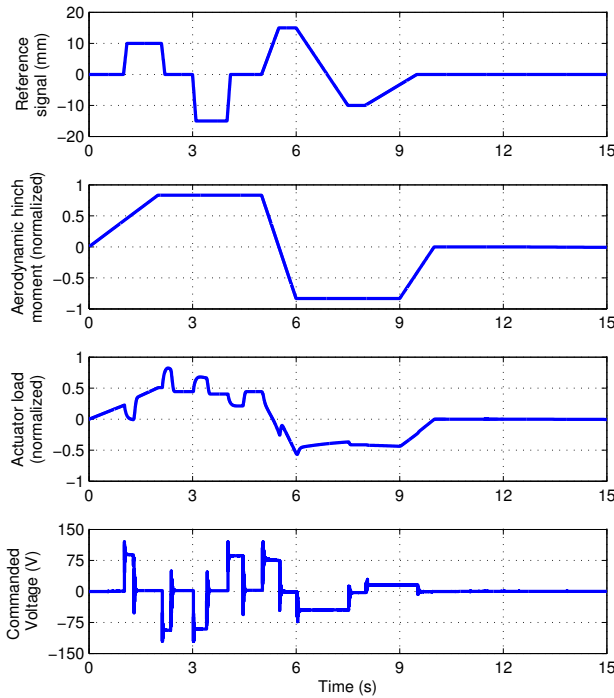

Fig. 4. Defined trajectories of reference input/aerodynamic hinge moment plus closed loop simulation results
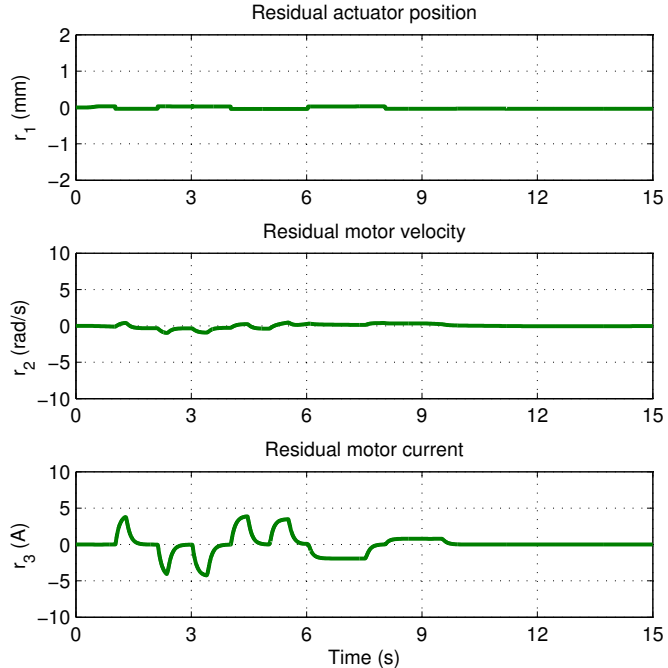

Fig. 5. Residual signals during the fault free simulation

appears in the residual without delay, in the remaining two cases a first order lag behavior with a time constant of $1 / 10$ s can be noticed. Overall the designed fault detection filter shows a satisfactory performance regarding the ability to detect and isolate the three different faults.

Another aspect worth mentioning at this point is, that fault detection and isolation generators to monitor sensors which are used for the purpose of control have to be designed using the open loop system model of the underlying system, i.e., without the controller dynamics. As during the normal operation of the EMA the sensor signals are directly fed back to the controller, the controller will try to alleviate occurring faults. Thus, these faults are not stationary detectable by considering the input output behavior of the closed loop system, although the system 

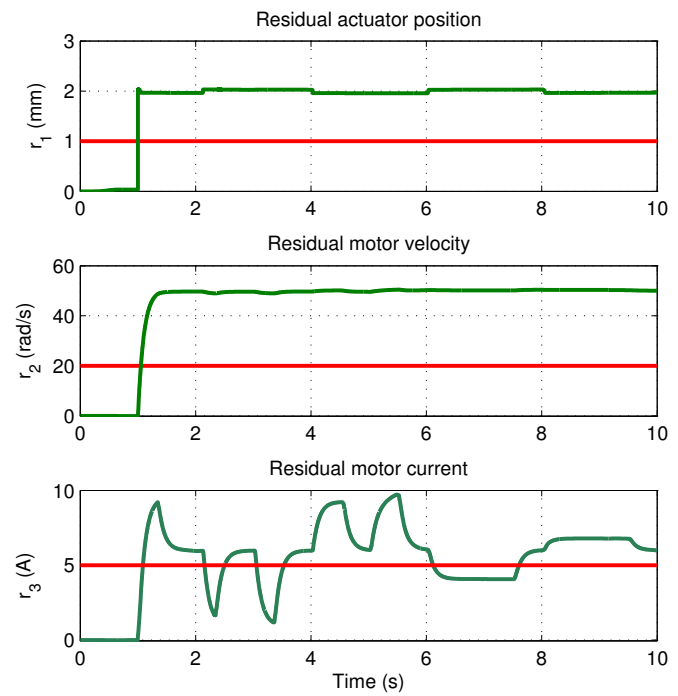

Fig. 6. Residuals during fault simulations

itself will show faulty state values. This is demonstrated in the example of Fig. 7: The plots show a simulation of a fault on the actuator position sensor. For a better view, the reference and disturbance inputs are set to zero. The fault is suppressed in the sensor signal after a short time and the sensor output is equal to the reference signal, namely zero making the fault stationary undetectable when considering the closed loop input output behavior. However, the actual actuator position is $-2 \mathrm{~mm}$. The residual signal generated by the first filter indicates this offset correctly (see first diagram Fig. 6), as it uses the signals demanded by the controller. What happens physically in the closed actuator loop in this fault situation is, that an additional input voltage is demanded by the controller to suppress the fault. This demanded voltage is fed also to the input of the residual filter, lifting the residual correctly to the fault amplitude.
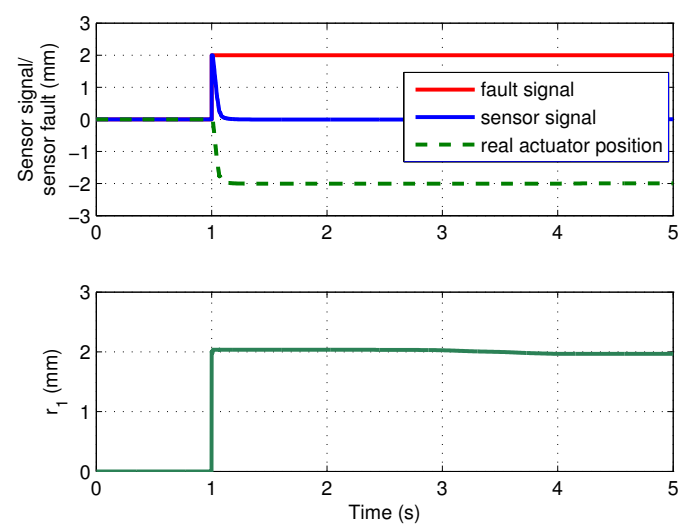

Fig. 7. Closed loop fault simulation results

\section{CONCLUSIONS}

The paper has considered the problem of sensor fault detection and isolation in electro-mechanical actuators which are used more and more in today's modern civil aircraft. A fault detection and isolation generator, designed by applying advanced nullspace computation techniques, to monitor three sensors relevant for the control of the actuator has been proposed. Such a residual generator can be used to detect small sensor fault in the electro-mechanical actuator and pave the way for different adaptation approaches in case of sensor malfunctions, as fault tolerant control or virtual sensor usage. The designed residual generator has been implemented and tested on a nonlinear high-fidelity simulation model of an electro-mechanical actuator, enabling a realistic validation. The residual generator adequately solves the fault detection and isolation problem as well as shows robustness against model uncertainties in the fault free cases.

\section{ACKNOWLEDGMENT}

This work was performed in the framework of the German LuFo V-1 EMA PFC Subsystem Project: LUFOV1-549-022.

\section{REFERENCES}

[1] P. Goupil, "Oscillatory failure case detection in the A380 electrical flight control system by analytical redundancy" Control Engineering Practice, vol. 18, pp. 1110-1119, 2010.

[2] A. Varga and D. Ossmann, "LPV-techniques based robust diagnosis of flight actuator faults," Control Engineering Practice, vol. 31, pp. 135147, 2014.

[3] B. Vanek, A. Edelmayer, Z. Szab, and J. Bokor, "Bridging the gap between theory and practice in lpv fault detection for flight control actuators," Control Engineering Practice, vol. 31, p. 171182, 2014.

[4] H. Alwi and C. Edwards, "Second order sliding mode observers for the addsafe actuator benchmark problem," Control Engineering Practice, vol. 31, p. 7491, 2014.

[5] E. A. Garcia, A. Zolghadri, P. Goupil, L. Lavigne, and P. Simon, "Nonlinear observer-based OFC detection for A380 aircraft," in Proc. of 7th IFAC Symposium on Fault Detection, Supervision and Safety for Technical Processes, (Barcelona, Spain), 2009.

[6] D. Henry, J.Cieslak, A.Zolghadri, and D.Efimov, "A non-conservative $H_{-} / H_{\infty}$ solution for early and robust fault diagnosis in aircraft control surface servo-loops," Control Engineering Practice, vol. 31, p. 183199, 2014.

[7] A. Marcos, "Application of H-infinity fault diagnosis to ADDSAFE benchmark: the control surface jamming case," in Proc. of AIAA Guidance, Navigation and Control Conference, (Portland, Oregon, USA), 2011.

[8] B. Vanek, Z. Szabo, A. Edelmayer, and J. Bokor, "Performance Comparison of Geometric and $H_{\infty}$ Fault Detection Filter Design: A Commercial Aircraft Example," in Proc. of 8th IFAC Symposium on Fault Detection, Supervision and Safety for Technical Processes, (Mexico City, Mexico), 2012.

[9] L. V. Eykeren, Q. Chu, and J. Mulder, "Actuator Fault Detection by Aerodynamic Model Identification," in Proc. of 8th IFAC Symposium on Fault Detection, Supervision and Safety for Technical Processes, (Mexico City, Mexico), 2012.

[10] D. Ossmann, "Enhanced fault detection and isolation in modern flight actuators," in Proc. of 3rd. Australian Control Conference, (Perth, Australia), 2013.

[11] M. Todeschi and L. Baxerres, "Airbus - health monitoring for the flight control emas 2014 status and perspectives," in Proc. of Recent Advances in Aerospace Actuation Systems and Components, 2014.

[12] E. Gilson, J.-D. Kopp, and D. Manzanares, "Moog next generation control and actuation," in Proc. of Recent Advances in Aerospace Actuation Systems and Components, 2014. 
[13] L. Romeral, J. Rosero, A. G. Espinosa, J. Cusido, and J. Ortega, "Electrical monitoring for fault detection in an ema," IEEE Aaerospace and Electronic Systems Magazine, vol. 25, pp. 4-9, 2010.

[14] C. Byington, M. Watson, D. Edwards, and P. Stoelting, "A modelbased approach to prognostics and health management for flight control actuators," in Proc. of IEEE Aerospace Conference, 2004.

[15] A. Garcia, J. Cusido, J. Rosero, and L. Romeral, "Reliable electromechanical actuators in aircraft," IEEE Aaerospace and Electronic Systems Magazine, vol. 23, pp. 19-25, 2008.
[16] A. Varga, "On computing achievable fault signatures," in Proc. of 7th IFAC Symposium on Fault Detection, Supervision and Safety for Technical Processes, (Barcelona, Spain), 2009.

[17] A. Varga, "On computing least order fault detection filters using rational nullspace bases," in Proc. of 5th IFAC Symposium on Fault Detection, Supervision and Safety for Technical Processes, (Washington D.C., USA), 2003.

[18] E. Frisk and M. Nyberg, "A minimal polynomial basis solution to residual generation for fault diagnosis in linear systems," Automatica, vol. 37, pp. 1417-1424, 2001. 